\title{
AiKomPass - Ein Instrument zur Sichtbarmachung informellen Lernens in der Metall- und Elektroindustrie
}

\author{
Martin Fischer $\mathbb{D} \cdot$ Magdalene Follner • Ines Rohrdantz-Herrmann • \\ Cüneyt Sandal
}

Eingegangen: 1. Juni 2018 / Angenommen: 3. Dezember 2018 / Online publiziert: 18. Dezember 2018 (C) Der/die Autor(en) 2018

Zusammenfassung AiKomPass ist ein webbasiertes Instrument, mit dessen Hilfe informelle und non-formale Kompetenzen aus dem Bereich der Metall- und Elektroindustrie sichtbar gemacht werden können. Das Instrument beruht auf einer Interviewstudie mit potenziellen Nutzern und auf einem Aufgabeninventar, welches Aufgaben aus den Arbeitsfeldern Arbeitsvorbereitung, Produktion, Instandhaltung und Logistik umfasst. Das Instrument selbst wurde in einem Prozess partizipativer Technikgestaltung entwickelt. Es hilft Arbeitskräften und Arbeitssuchenden, ihre Fähigkeiten zur Bewältigung von Arbeitsaufgaben selbst einzuschätzen und visualisiert auf diese Weise das hierfür erforderliche Wissen und Können. Überdies dient es zur Analyse biografischer Aspekte von Kompetenzen (außerhalb der Erwerbsarbeit und außerhalb der Metall- und Elektroindustrie angeeignete Kompetenzen sowie relevante Zertifikate). Das Online-Tool richtet sich besonders an Menschen, denen formale Qualifikationen für den Arbeitsmarkt fehlen. AiKomPass ist online verfügbar (www.aikompass.de) und kann kostenlos verwendet werden.

Prof. Dr. M. Fischer $(\bowtie) \cdot$ M. Follner, M.A. · I. Rohrdantz-Herrmann, M.A.

Karlsruher Institut für Technologie (KIT), Universität des Landes Baden-Württemberg und nationales Forschungszentrum in der Helmholtz-Gemeinschaft, Hertzstr. 16, Geb. 06.41, 76187 Karlsruhe, Deutschland

E-Mail: m.fischer@kit.edu

M. Follner, M.A.

E-Mail: magdalene.follner@kit.edu

I. Rohrdantz-Herrmann, M.A.

E-Mail: rohrdantz@kit.edu

C. Sandal, M.A.

Duale Hochschule Baden-Württemberg (DHBW), Karlsruhe, Deutschland

E-Mail: cueneyt.sandal@dhbw-karlsruhe.de 
Schlüsselwörter Arbeitserfahrung · Aufgabeninventar · Kompetenzen · Informelle $\cdot$ Metall- und Elektroindustrie $\cdot$ Validierung

\title{
AiKompass-A tool to make informal learning within the electrical and metal industries visible
}

\begin{abstract}
AiKomPass is a web-based tool to make informal and non-formal competences within the electrical and metal industries visible. It is based on an interview study with potential users and a task inventory, which covers tasks of work areas like process planning, production, maintenance and logistics within production processes. The tool itself was developed within a participative design process. It enables employees and people in search of employment to self-assess their ability to fulfil working tasks and thus visualizes their skills and knowledge. In addition it helps to analyse biographical aspects of competences (competences from spheres outside work and outside the electrical and metal industries and related certificates). The online tool focuses especially on people who are not formally qualified for the labour market. AiKomPass is available online (www.aikompass.de) and can be used free of charge.
\end{abstract}

Keywords Work experience - Task inventory $\cdot$ Competences $\cdot$ Informal $\cdot$ Metal and electrical industries · Validation

\section{Einleitung und Vorstellung der Forschungsfrage}

Die Anerkennung informell erworbener Kompetenzen setzt deren Dokumentation und Sichtbarmachung voraus. Welche Rolle können potenziell Anerkennungswillige hierbei einnehmen, und welches (webbasierte) Instrument eignet sich dafür? Dies war die Forschungsfrage, die sich in zwei öffentlich geförderten Forschungsund Entwicklungsprojekten (FuE) stellte, welche sich ab dem Jahr 2012 dem Bereich der Metall- und Elektroindustrie in Baden-Württemberg widmeten. Damit war dies seinerzeit einer der ersten praktischen Ansätze, die sich mit der Validierung von informell und non-formal erworbenen Kompetenzen befassten. Der Ansatz war jedoch nur auf eine Branche und ein deutsches Bundesland bezogen und verblieb noch jenseits formaler Validierungs- und Anerkennungsverfahren.

Die FuE-Projekte gingen von der Prämisse aus, dass es einen relevanten Teil von Arbeitskräften gibt, die über keinen formalen Abschluss verfügen und deshalb Schwierigkeiten haben, ihre Kompetenzen nachzuweisen, wenn ihre Arbeitsplätze in Gefahr geraten und wenn sie sich beruflich umorientieren wollen oder müssen. In Baden-Württemberg waren dies immerhin 14,4\% aller abhängig Beschäftigten das macht etwa 600.000 Personen aus (Südwestpresse 2014). Auch für Unternehmen, die über Fachkräftemangel klagen, ist es schwierig, kompetente Arbeitskräfte (innerhalb und außerhalb des Unternehmens) überhaupt wahrzunehmen, wenn diese die entsprechenden Zertifikate nicht besitzen. Die skizzierte Problematik betrifft nun nicht nur Personen, die über keinerlei formalen Abschluss verfügen; sie betrifft auch Menschen, die nicht mehr im erlernten Beruf arbeiten (nur 55\% der Auszubil- 
denden verbleiben nach Ausbildungsabschluss in ihrem Beruf (Schier 2011, S. 9)), und sie betrifft Personen, die in Arbeitsbereichen wie der betrieblichen Instandhaltung oder der betrieblichen Arbeitsvorbereitung arbeiten, die keinem bestimmten Berufsabschluss der beruflichen Erstausbildung in Deutschland entsprechen. Daher widmeten sich die FuE-Projekte den Arbeitsbereichen der Produktion und Logistik, wo besonders viele An- und Ungelernte arbeiten, sowie der Instandhaltung und Arbeitsvorbereitung, wo zwar meist Fachkräfte tätig sind, jedoch ohne einen für den Arbeitsbereich spezifischen Abschluss.

\section{Vorstellung der Projekte zur Sichtbarmachung informellen Lernens in der Metall- und Elektroindustrie}

Mit der Einführung des Deutschen Qualifikationsrahmens (DQR) sowie der Empfehlung des Rates der Europäischen Union, bis spätestens 2018 Verfahren zur Validierung nicht-formalen und informellen Lernens zu implementieren, ist die Frage der Anerkennung informell erworbener Kompetenzen auch in Deutschland politisch auf die Agenda gesetzt worden. In den Projekten Anerkennung informell erworbener Kompetenzen in der Metall- und Elektroindustrie (AiKo) und Anerkennung informell erworbener Kompetenzen im Bereich der Lagerlogistik und produktionsnahen Logistik (AiKo-ProdLog) wurde diese Thematik näher in den Blick genommen.

Projektträger war die AgenturQ, eine gemeinsame Qualifizierungsagentur der IG Metall Baden-Württemberg und des Arbeitgeberverbandes Südwestmetall nach dem Tarifvertrag zur Qualifizierung. Die wissenschaftliche Begleitung im Projekt AiKo oblag dem Karlsruher Institut für Technologie sowie der Pädagogischen Hochschule Heidelberg. Im Rahmen der Projektlaufzeit wurde sowohl der Bedarf für die Anerkennung informell erworbener Kompetenzen im Bereich der Metall- und Elektroindustrie $(\mathrm{M}+\mathrm{E})$ erhoben als auch ein Instrument für die Anerkennung von informell erworbenen Kompetenzen entwickelt. Im Fokus der Projekte standen anund ungelernte Beschäftigte und die Gruppe der Facharbeiterinnen und Facharbeiter. Für beide Zielgruppen wurde empirisch erhoben, welche informell erworbenen Kompetenzen relevant sind und wie diese Kompetenzen mithilfe des Instruments AiKomPass sichtbar gemacht werden können.

Das Projekt AiKo (gefördert vom Ministerium für Finanzen und Wirtschaft in Baden-Württemberg im Rahmen der „Allianz für Fachkräfte“, Laufzeit von Juli 2012 bis April 2015) hat sich mit der Sichtbarmachung von informell und nonformal erworbenen Kompetenzen in der Metall- und Elektroindustrie befasst. Das Folgeprojekt AiKo-ProdLog (gefördert vom Ministerium für Wirtschaft, Arbeit und Wohnungsbau Baden-Württemberg, Laufzeit von Januar 2016 bis März 2017) knüpft hieran an und erweitert die zugrunde liegenden Arbeitsaufgaben aus den Arbeitsbereichen Arbeitsvorbereitung, Instandhaltung und Produktion um den Bereich der Lagerlogistik und produktionsnahen Logistik, sodass alle wesentlichen Arbeitsbereiche für gewerbliche Arbeitskräfte in der Metall- und Elektroindustrie abgedeckt sind. Das webbasierte Instrument AiKomPass dient unter anderem dazu, Fachkräftepotenziale ausfindig und für den Arbeitsmarkt in Baden-Württemberg nutzbar zu machen. Das Instrument beruht im Wesentlichen auf einem Aufgabeninventar-einer 
strukturierten Darstellung der in der Fertigung in der M+E-Industrie vorfindlichen Arbeitsaufgaben (Frieling et al. 2000). Die Nutzer des Instruments können angeben, inwieweit sie die dort vorgegebenen Aufgaben in den Arbeitsfeldern Arbeitsvorbereitung, Produktion, Instandhaltung und Produktions- und Lagerlogistik beherrschen und auf dieser Basis ein persönliches Kompetenzprofil erstellen.

Eine wesentliche Erweiterung besteht darin, dass über AiKomPass nicht nur die typischen Arbeitstätigkeiten erhoben werden, sondern auch Kompetenzen, die sich aus Aktivitäten aus dem Freizeitbereich entwickeln. Die im Rahmen der Projekte durchgeführte Interviewstudie hat ergeben, dass wesentliche überfachliche Kompetenzen eher außerhalb der Arbeit erworben werden - etwa durch ehrenamtliches Engagement, Hobbys oder in der Familie. So gaben beispielsweise die Befragten an, Kompetenzen im Bereich der Teamarbeit oder bei der Organisation von Gruppen im Kontext von Aktivitäten bei Vereinen, der Freiwilligen Feuerwehr oder des Technischen Hilfswerks erworben zu haben.

Die Anerkennung informell erworbener Kompetenzen ist nicht nur eine Frage der sachgerechten Abbildung von Kompetenzen, sondern auch eine Frage der gesellschaftlichen Akzeptanz. In dieser Hinsicht ist in den Projekten ein Verfahren der partizipativen Technikentwicklung (vgl. Fischer 2000, S. $249 \mathrm{ff}$.) erarbeitet und erprobt worden - unter Einbeziehung von Sozialpartnern, Personalverantwortlichen, Betriebsräten, Kammern, Arbeitsvermittlern, Wissenschaftlern und Betroffenen, das gleichermaßen zur Praktikabilität wie auch zur gesellschaftlichen Akzeptanz des Instruments AiKomPass beigetragen hat. Dieses Konzept sieht vor, dass künftigen Nutzern, Personalverantwortlichen, Betriebsräten u. a. zwar durchaus ein Vorschlag für die Ausgestaltung des Instruments auf Basis der wissenschaftlichen Analyse vorgelegt worden ist (weil die Nutzer auch nicht ad hoc sagen könnten, wie solch ein Instrument aussehen soll). Die endgültige Gestalt und damit die Gebrauchstauglichkeit des Instruments entstanden jedoch vor allem durch die Diskussion und Erprobung von Prototypen des Instruments durch potenzielle Nutzer.

\section{Informell erworbene Kompetenzen}

Es ist eine allgemein geteilte Annahme, dass viele Kompetenzen - und besonders arbeitsrelevante Kompetenzen - durch Lernen im Prozess der Arbeit erworben werden (Boreham et al. 2002; Fischer et al. 2004). Dem erfahrungsgeleiteten Lernen im Arbeitsprozess wird auch und gerade im Kontext computergestützter Arbeit große Bedeutung beigemessen (Böhle und Rose 1992; Bauer et al. 2002) - also dort, wo arbeitsimmanentes Lernen aufgrund der Komplexität von Arbeitsumgebungen, der Intransparenz technischer Phänomene und Prozesse sowie des Zeitdrucks vermutlich besonders schwierig ist, um es vorsichtig auszudrücken. Es scheint also paradox, worauf schon Antonius Lipsmeier (1996, S. 209) hingewiesen hat, dass dem Erfahrungslernen im Prozess der Arbeit immer noch ein solch hoher Stellenwert zukommt.

Es gibt trotzdem mindestens einen gewichtigen Grund, Erfahrung für die Entwicklung beruflicher Kompetenz, insbesondere beruflichen Könnens, für unverzichtbar zu erachten - dieser ist die Authentizität des Erfahrungslernens (vgl. Fischer 2000). 
Die Echtheit der Erfahrung gewährleistet, dass der Möglichkeit nach alle Facetten einer Situation in die persönliche Erfahrung einfließen können. Diese Facetten können redundant sein (Siebeck 1994, S. 112): Es werden auch zufällige, für die Situation eigentlich nicht wesentliche Erscheinungen in die persönliche Erfahrung integriert. So registriert zum Beispiel ein Autofahrer möglicherweise die Kleidung von Fußgängern, was für das Autofahren an sich unwesentlich ist. Die gesamte Erscheinungsvielfalt aufzunehmen und zu verarbeiten, ist jedoch dort besonders wichtig, wo eine Situation oder ein Prozess nicht nur theoretisch auf den Begriff gebracht, sondern auch praktisch beherrscht werden muss, und wo bei der Prozessbeherrschung vielfältige Phänomene eine Rolle spielen. Sicherlich ist für das Autofahren, um dieses Beispiel noch einmal aufzugreifen, die Kenntnis der Verkehrszeichen und Verkehrsregeln wichtig. Die Beherrschung des Autofahrens gelingt jedoch nur auf Basis des Selbst-Machens von Erfahrung, da beim Vorankommen nicht nur allgemeine Regeln und Bedeutungen, sondern die Phänomene des Konkreten (das Verhalten der übrigen Verkehrsteilnehmer, das Verhalten des eigenen Autos etc.) berücksichtigt werden müssen. Und so gelingt auch die Beherrschung von Maschinen, das Hüten von Kindern oder die Pflege kranker Menschen nur auf Basis von Erfahrung, da immer auch der konkrete Zustand von Objekten und Personen in spezifischen Situationen wahrgenommen und berücksichtigt werden muss.

Dieser konkrete Zustand von Objekten und Personen in spezifischen Situationen ist nun in der Arbeitswelt gar nicht immer eindeutig - er muss interpretiert, mit Schlussfolgerungen versehen, schließlich bewältigt bzw. verändert werden und schafft so einen Lernanlass. Bei der gewerblich-technischen Arbeit ist dieser Lernanlass häufig eine Problemsituation: eine Maschinenstörung, ein Lieferengpass, Personalknappheit oder ein Eilauftrag. Was dabei wichtig ist, hat man in Deutschland im Rahmen der Kritischen Psychologie (z. B. Holzkamp 1993) ganz ähnlich gesehen wie in den USA Donald Schön, der das Handeln von professionellen Praktikern analysiert hat. Schön kommt zu folgendem Ergebnis:

\begin{abstract}
From the perspective of Technical Rationality professional practice is a process of problem solving. Problems of choice or decision are solved through the selection, from available means, of the one best suited to established ends. But with this emphasis on problem solving, we ignore problem setting, the process by which we define the decision to be made, the ends to be achieved, the means which may be chosen. In real-world practice, problems do not present themselves to the practitioner as given. They must be constructed from the materials of problematic situations which are puzzling, troubling and uncertain. In order to convert a problematic situation to a problem, a practitioner must do a certain kind of work. He must make sense of an uncertain situation that initially makes no sense (Schön 1983, S. 39f.).
\end{abstract}

Bei der Bewältigung von Problemsituationen tritt ein weiteres Moment hinzu, das für das Lernen im Arbeitsprozess relevant ist, nämlich Lernen in sozialer Interaktion. In einer Studie, die von Michael Eraut et al. (1998) in Großbritannien durchgeführt wurde, nannten die 120 Befragten aus dem Maschinenbau, aus dem Bereich Wirtschaft und Verwaltung sowie aus dem Gesundheitssektor vor allem 
zwei Ressourcen für das Lernen im Arbeitsprozess. Erstens: „The challenges of work itself“. Und zweitens: „Learning from other people“.

Eraut und Mitarbeiter gelangen zu folgendem Resümee:

The outcome of such "learning from experience" was the development of knowledge, skills and understanding, although this was difficult to explain to others. Effective learning was, however dependent on confidence, motivation and capability - prerequisites for employees' selfmanagement of much of their learning (Eraut et al. 1998).

Aus dem Gesagten ergibt sich für die Erfassung informell erworbener Kompetenzen Folgendes: „Making collective sense of events in the workplace“ (Boreham 2004, S. 3) ist der Stimulus, der zur Aneignung von Kompetenzen im Prozess der Arbeit führen kann. Über Routinetätigkeiten hinaus erwerben die Beschäftigten dann durch die Bewältigung von Problemsituationen in Kooperation mit Kollegen (im günstigen Fall) Wissen und Können, welches für die Arbeit bedeutsam ist. Die Ergebnisse solchen Lernens sind jedoch in Deutschland in der Regel nirgendwo festgehalten - sieht man einmal von den formelhaften Beschreibungen in Arbeitszeugnissen ab. Es ist daher davon auszugehen, dass es einen großen Anteil von Menschen gibt, die Kompetenzen für die Erwerbsarbeit durch Lernen im Arbeitsprozess erworben haben, solche Kompetenzen aber mangels formaler Abschlüsse nicht nachweisen können.

Informelle, non-formale und formale Kompetenzen sind jedoch schwierig voneinander abgrenzbar. Dieselbe Kompetenz, z. B. die Beherrschung einer bestimmten Maschine, kann informell (z. B. durch ,Abgucken“ von Kollegen), non-formal (z. B. im Rahmen eines Kurses) oder formal (z. B. im Rahmen einer beruflichen Erstausbildung) angeeignet worden sein. Und auch in formalen Settings werden Kompetenzen informell erworben, z. B. Fähigkeiten zur Teamarbeit, wenn in Gruppen gelernt wird, oder Fremdsprachenkenntnisse, wenn in der Fachausbildung englische Begriffe verwendet werden oder englischsprachige Fachlektüre.

Formales, informelles und non-formales Lernen sind daher keine vollkommen voneinander abgrenzbaren Merkmale, sondern Attribute, die dem Lernen zugeschrieben werden (Colley et al. 2003). Daraus folgt: Will man informell erworbene Kompetenzen erfassen, kann man aus dem Spektrum der relevanten Kompetenzen von vornherein nichts ausschließen.

Bei der Bestimmung informellen Lernens bzw. informell erworbener Kompetenzen wird immer wieder die Frage aufgeworfen, ob informelles Lernen bewusst und intentional oder aber eher beiläufig - implizit und nicht beabsichtigt - stattfindet. Institutionen wie die OECD (OECD 2006, S. 4) oder CEDEFOP (2009, S. 74) betonen die Nicht-Intentionalität informellen Lernens, während in der regelmäßig durchgeführten Weiterbildungsbefragung Adult Education Survey (AES) als informelles Lernen ausschließlich bewusst beabsichtigtes Lernen erfasst wird (vgl. Baethge et al. 2010, S. 168). Von der Sache her gibt es wenig Grund anzunehmen, dass informelle Kompetenzen weder ausschließlich beiläufig, ohne bewussten Entschluss, noch ausschließlich nur als Folge eines bewussten Entschlusses angeeignet worden sind. Untersuchungen zum Lernen im Arbeitsprozess (z.B. Fischer und Röben 2004) haben ergeben, dass Fachkräfte sich öfter um Informationen über die bei ihrer Ar- 
beit einzusetzenden Werkzeuge und Verfahren (z. B. Handreichungen, Handbücher, Fachartikel) bemühen und bisweilen ihre Arbeitserfahrungen dokumentieren: So ist es im Hafen von Antwerpen Tradition, dass die Arbeitskräfte kleine graue Kladden mit sich führen, in denen sie persönliche Arbeitserfahrungen, Regeln, Anweisungen etc. notiert haben (Fischer und Röben 2004). Aber: Wer liest schon unbeabsichtigt Bücher oder schreibt unbewusst seine Erfahrungen auf? Andererseits: Wenn unter Zeitdruck eine defekte Maschine zu reparieren ist und bei der Fehlerbehebung notgedrungen Lernprozesse zu absolvieren sind, die eben dann beiläufig passieren wer hat denn schon in solch einer Situation die Absicht formuliert: Jetzt möchte ich aber etwas dazulernen?

Es gibt also weder Grund, Lernintentionen aus dem informellen Lernen auszuklammern noch solche Intentionen für unbedingt notwendig zu halten; aber es gibt Anlass zu der Annahme, dass den Individuen eine Verbalisierung ihrer durch informelles Lernen angeeigneten Kompetenzen schwerfällt, erst recht eine objektive, eindeutige und vergleichbare Benennung von Kompetenzen. Gleichgültig, ob Kompetenzen durch Lernen im Prozess der Arbeit oder in institutionellen Lernumgebungen erworben worden sind, immer erfordert es eine gesonderte Reflexion darüber, welche Art von Können aus dem jeweiligen Lernprozess entstanden ist. Wenn also die Subjekte es selber sind und sein sollen (wie in unseren Projekten), die ihr Können dokumentieren und sichtbar machen, dann muss ein Rahmen vorgegeben werden, der zugleich einen Stimulus für die Reflexion und eine Struktur für die Einordnung bietet. Diesen Rahmen bietet ein Aufgaben- bzw. Tätigkeitsinventar - eine möglichst vollständige und strukturierte Liste von Tätigkeiten in einem Aufgabengebiet, anhand derer sich Menschen fragen können: Beherrsche ich diese Tätigkeit oder nicht?

\section{Methodisches Vorgehen}

In den Projekten AiKo und AiKo-ProdLog wurde das Kompetenzerfassungsinstrument AiKomPass für die Metall- und Elektroindustrie entwickelt. Das Aufgabeninventar wurde sukzessive anhand von Dokumentenanalysen, leitfadengestützten Interviews und Arbeitsplatzbeobachtungen erstellt und von betrieblichen Experten validiert.

Das verwendete Aufgabeninventar basiert auf einer strukturierten Aufgabenliste für industrielle Produktionstätigkeiten (u.a. aus einem Projekt des Fraunhofer Instituts (IAO) Stuttgart, vgl. Witzgall 2009) und wurde für die Anforderungen in den Projekten weiterentwickelt und inhaltlich erweitert. Dabei wurden auch Ausbildungsordnungen und Rahmenlehrpläne relevanter Berufe (wie bspw. „Fachkraft für Lagerlogistik“) sowie (betriebliche) Qualifizierungsprofile aus vorangegangenen Weiterbildungsprojekten (WAP - Weiterbildung im Prozess der Arbeit, vgl. Bauer et al. 2007) verwendet. Weiterhin wurde Bezug genommen auf curriculare Inhalte von Einstiegsqualifizierung (EQ) auf betrieblicher Ebene, Weiterbildungsangebote insbesondere für An- und Ungelernte. Zudem wurden Stellenanzeigen in verschiedenen Onlineportalen durchsucht, um auch Anforderungen auf betrieblicher Ebene 
zu eruieren. Auch Standards wie DIN-Normen sowie das Entgeltrahmenabkommen (ERA-TV) wurden berücksichtigt.

Zur Identifizierung von Arbeitsaufgaben in der Metall- und Elektroindustrie wurden ergänzend eine Interviewstudie und Arbeitsplatzbeobachtungen durchgeführt. Im Rahmen des Projektes wurden 75 Interviews in 10 Betrieben durchgeführt. Bei der Analyse von Arbeitstätigkeiten standen vor allem die An- und Ungelernten, aber auch die gelernten Fachkräfte im Fokus. Weiterhin wurden auch fachliche Vorgesetzte interviewt, soweit sie Angaben zu Art, Umfang und Systematisierung der relevanten Arbeit machen konnten. Es wurde darauf Wert gelegt, Personen in die Studie einzubeziehen, die aktiv an den Arbeitstätigkeiten beteiligt sind, also diese entweder selbst durchführen oder aber Vorgesetzte der Durchführenden sind. So erfolgte mittels Beobachtungsinterviews nach der Methode der BAG-Analyse (Analyse beruflicher Arbeitsaufgaben; siehe Reinhold et al. 2003) eine Analyse und Beschreibung von Arbeitssituationen in den zu untersuchenden Bereichen. Ziel war es, Geschäfts- und Arbeitsprozesse, Handlungsschritte, konkrete Arbeitsaufgaben, Merkmale des Arbeitsprozesses wie eingesetzte Werkzeuge und Methoden sowie Organisationsformen der Arbeit, Anforderungen wie auch Schnittstellen zu anderen beruflichen Tätigkeiten aufzudecken. Außerdem wurden auch Mitarbeitende des Personalwesens, Führungskräfte und Betriebsräte eingebunden, da diese Personenkreise mit Personalentscheidungen betraut sind.

Zeitgleich wurden auch Verbände, (universitäre) Institutionen (z.B. im Bereich der Arbeitswissenschaften) und Vereine, Bildungsexperten sowie Vertreter der Sozialpartner IG Metall und Südwestmetall in den Prozess einbezogen.

Zur Validierung des Aufgabeninventars wurden Expertenbefragungen durchgeführt. Auf diese Weise erfolgte eine Evaluation und Akzeptanzprüfung des Aufgabeninventars, das dem zu entwickelnden Tool zugrunde gelegt werden sollte, hinsichtlich Aufbau und Praktikabilität wie auch hinsichtlich Aufgabenauswahl und der Möglichkeit, als Instrument der Kompetenzerfassung und -dokumentation zu fungieren. Dabei wurde das fachliche Aufgabeninventar zum einen betrieblichen Expertinnen und Experten vorgelegt mit der Bitte, es auf Vollständigkeit und Konsistenz zu prüfen. Zum anderen wurden, um die wissenschaftliche Perspektive zu berücksichtigen, Expertinnen und Experten aus dem Bereich der Wissenschaft in die Evaluation des Tools einbezogen. Hierdurch konnte das Tool AiKomPass nochmals verbessert werden. Die Rückmeldungen sowohl von den betrieblichen Experten als auch von den Wissenschaftlern waren durchweg positiv. Des Weiteren konnten in einer partizipativen Pretest-Phase interessierte Personen das Kompetenztool zur Anerkennung informell erworbener Kompetenzen im Bereich der Lagerlogistik und der produktionsnahen Logistik testen und damit die Gebrauchstauglichkeit des Tools optimieren helfen. Diese Tests wurden wissenschaftlich begleitet und dienten der Weiterentwicklung, Akzeptanzprüfung und Validierung des Aufgabeninventars. 


\section{Projektergebnisse}

\subsection{Tätigkeitsbasierte Darstellung von Arbeitsinhalten}

Über die Web-Anwendung AiKomPass können Nutzer aus einer strukturierten Liste diejenigen Aufgaben auswählen, die sie in der Lage sind auszuführen und die sie aktuell noch erledigen. Die Aufgaben stammen aus den Arbeitsfeldern Arbeitsvorbereitung, Produktion, Instandhaltung sowie Produktions- und Lagerlogistik. Dieses fachliche Aufgabeninventar ist erweitert um Tätigkeiten, die in der Freizeit von Bedeutung sind. Das Ergebnis der Nutzung ist ein individuelles Gesamtprofil, welches für die persönliche und berufliche Weiterentwicklung herangezogen und bei der Validierung informell erworbener Kompetenzen genutzt werden kann.

Die eigenständige Beschreibung von Kompetenzen erweist sich in vielen Situationen als besonders schwierig, weshalb eine professionelle Begleitung erforderlich erscheint (Fischer et al. 2014). Zudem konnte festgestellt werden, dass die Beschreibung der Kompetenzen mit Bezug zur Arbeitspraxis sowie den Veränderungen innerhalb der Arbeitspraxis die Reflexion über das erworbene Wissen und Können erleichtert.

Für die informationstechnologische Umsetzung bestand zudem der Anspruch, dass möglichst wenig eigene Formulierungen verwendet werden sollen. Insgesamt ergab sich hierdurch die Forderung, dass sich die Kompetenzabfragen an beruflichen Aufgaben und gleichzeitig an vorhandenen vorformulierten Aufgabenbeschreibungen orientieren sollten. Hierzu ist eine Zusammenstellung von für die Arbeitsfelder (Arbeitsvorbereitung, Produktion, Instandhaltung, Produktions- und Lagerlogistik) typischen Arbeitsaufgaben erforderlich, die dem Kompetenzerfassungsinstrument als Datenbasis zugrunde gelegt werden. Hieraus können Nutzer die für sie relevanten Aufgaben auswählen, sofern die Aufgabe im Rahmen der aktuellen Arbeitstätigkeit bearbeitet wird oder im Laufe des Arbeitslebens schon erfolgreich bearbeitet wurde.

In der Testphase konnten die Teilnehmer das Instrument für die Zusammenstellung der Aufgaben verwenden, die sie tatsächlich beherrschen. Die aufgabenorientierte Kompetenzerfassung hat sich im Rahmen der durchgeführten Untersuchungen für den Nutzerkreis als sehr hilfreich erwiesen. Die vorformulierten Arbeitsaufgaben sowie die Verwendung von arbeitstypischen Begrifflichkeiten führten zu einem hohen Wiedererkennungswert.

Fachexperten profitieren von aus Arbeitsinventaren zusammengestellten Aufgabenlisten gleichermaßen. So können bspw. Vorgesetzte und Personalentscheider hieraus Kompetenzen interpretieren und mit betrieblichen Anforderungsprofilen vergleichen.

Die Zusammenstellung von Aufgabeninventaren erfordert zum einen die Erfassung aller für den Untersuchungsbereich relevanten typischen Arbeitsaufgaben. Zum anderen müssen diese Aufgaben innerhalb einer Systematik strukturiert werden: Arbeitsaufgaben mit engerem Bezug werden zu Aufgabengruppen zusammengefasst, die wiederum mit anderen Aufgabengruppen zu Teilarbeitsfeldern zusammengeführt werden. Letztlich werden alle Teilarbeitsfelder unter einem gemeinsamen Arbeitsfeld angeordnet. 
Die Zusammenstellung von beherrschbaren Arbeitsaufgaben wird als Ergebnis innerhalb eines persönlichen Aufgabenprofils dargestellt. Dieses Aufgabenprofil ist Ergebnis einer Selbsteinschätzung. Für die Ableitung von validierten Kompetenzen bedarf es daher einer weitergehenden Analyse und Interpretation von Experten aus der Praxis.

Bei der Nutzung des Instruments entstehen individuelle Aufgabenprofile, die zum einen sehr detaillierte Auskunft über die Arbeitserfahrungen der Nutzer geben und zum anderen durch ihre Strukturierung und die Vollständigkeit des zugrunde liegenden Aufgabeninventars auch einen Vergleich mit Kompetenzanforderungen (z.B. Ausbildungsprofil/-ordnung) ermöglichen. Die Verbindung zwischen der Darstellung als Aufgabenprofil und den vorhandenen Kompetenzen wird jedoch nicht direkt durch das Instrument hergestellt, sondern bedarf der Interpretation/Bewertung durch Expertinnen und Experten aus der Praxis.

\subsection{Die Webanwendung AiKomPass}

Das im AiKomPass zu erstellende Profil wird aus mehreren Bereichen erstellt, durch die die Nutzer strukturiert geführt werden. Alternativ können die einzelnen Bereiche auch in beliebiger Reihenfolge bearbeitet werden. Hierzu steht eine Navigation zur Verfügung, welche das beliebige Wechseln ermöglicht. In den einzelnen Bereichen stehen unterschiedliche Felder zur Verfügung, die der Nutzer bei Bedarf bearbeiten kann; auf Pflichtfelder wurde explizit verzichtet (Abb. 1).

AiKomPass erfasst in dem Bereich Lebenslauf einzelne Stationen des Arbeitslebens. So können betriebliche Sonderaufgaben, Schulungen für berufliche Tätigkeiten wie auch ehrenamtliche Tätigkeiten aufgeführt werden.

Die wichtigste Funktion des Instruments AiKomPass ist die Zusammenstellung von Arbeitsaufgaben aus dem Bereich Metall und Elektro, die die Person im Arbeitsleben schon erfolgreich bearbeitet hat und/oder immer noch bearbeiten kann. Alle im AiKomPass zusammengestellten Arbeitsaufgaben sind Aufgabengruppen zugeordnet. Die Aufgabengruppen wiederum werden in Teilarbeitsfeldern zusammengefasst. Die Teilarbeitsfelder werden den vier Arbeitsfeldern Arbeitsvorbereitung, Produktion, Instandhaltung sowie Produktions- und Lagerlogistik zugeordnet:

Der Nutzer beginnt mit der Auswahl der relevanten Arbeitsfelder. Im zweiten Schritt erscheinen die entsprechenden Teilarbeitsfelder, wie bspw. Produktionsaufträge einplanen in der Arbeitsvorbereitung, Fertigen in der Produktion oder Wartung in der Instandhaltung (Abb. 2).

Im dritten Schritt erscheinen nun die Aufgabengruppen der ausgewählten Teilarbeitsfelder. Nach der Auswahl der Aufgabengruppen können die hierauf bezogenen Aufgaben ausgewählt werden. Diese werden in einer Gesamtübersicht dargestellt.

Berufserfahrungen außerhalb der Metall- und Elektroindustrie können in dem Bereich Nicht-fachliches Aufgabenprofil außerhalb $M+E$ zusammengestellt werden. Hier werden nicht nur die Tätigkeiten erfasst, sondern auch das Wissen und Können, das dabei erworben wurde.

Der Nutzer hat des Weiteren die Option, seine Sprach-und Computerkenntnisse sowie seine Kompetenzen aus Freizeitaktivitäten und ehrenamtlichen Tätigkeiten zu erfassen. 


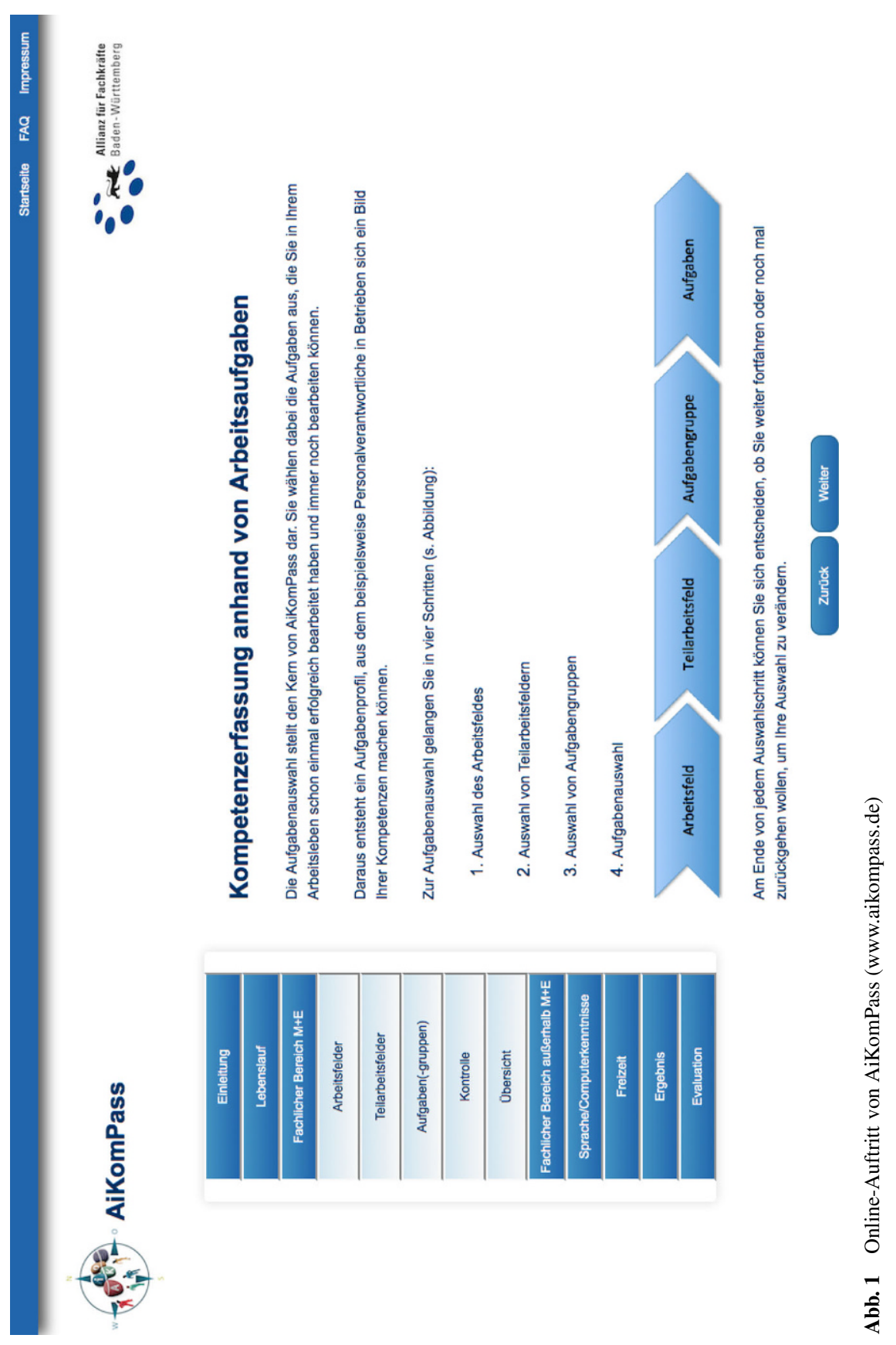



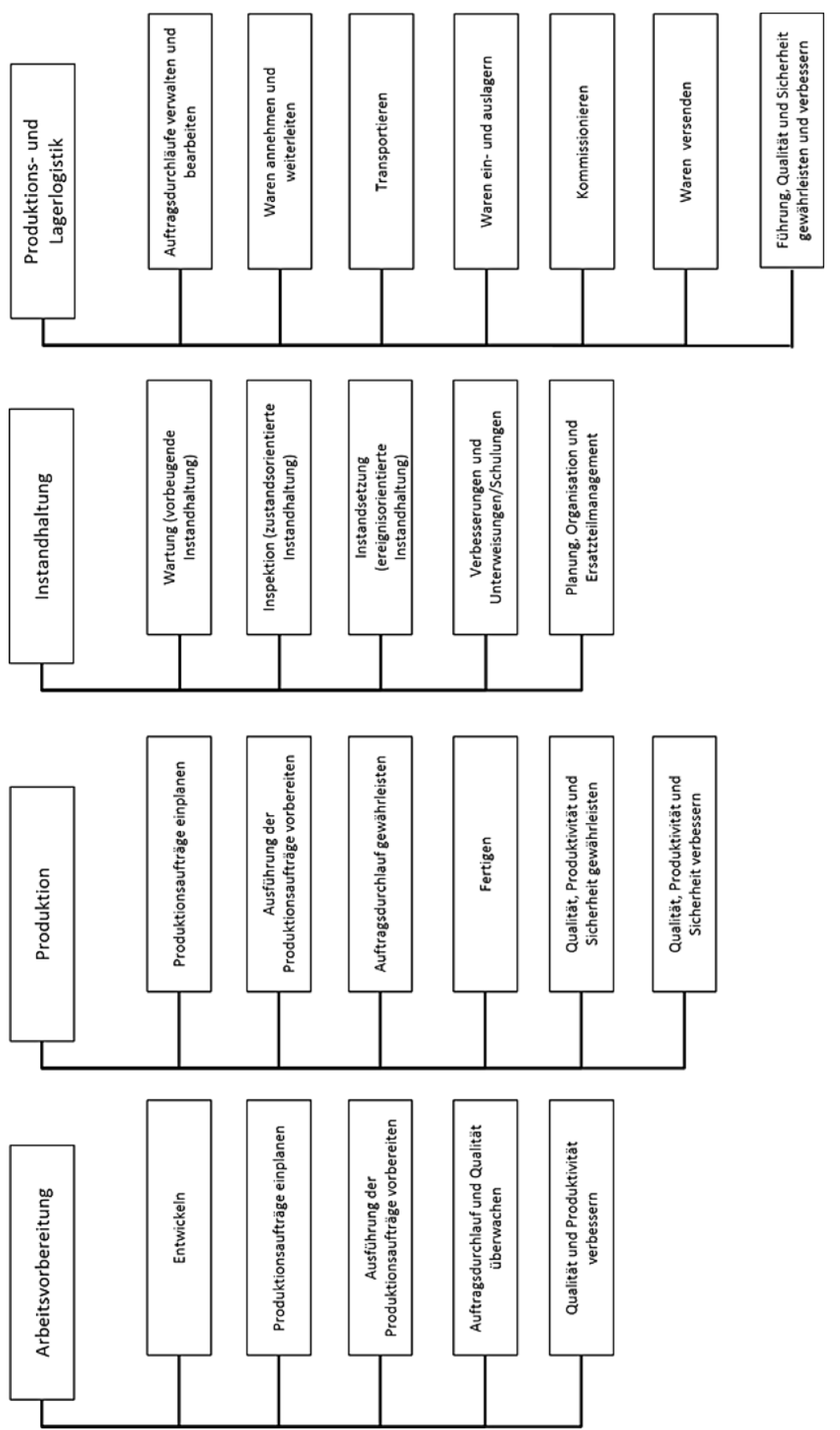

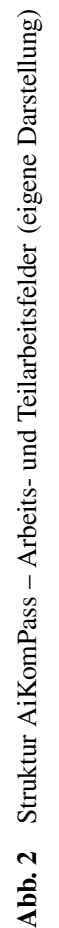




\section{Nutzungsmöglichkeiten von AiKomPass}

Das Instrument AiKomPass ist im Internet für alle frei verfügbar. Über www. aikompass.de gelangen Interessierte auf die Informationsseiten rund um die Projekte AiKo und AiKo-ProdLog und können von dort AiKomPass starten.

Das PDF-Dokument als Ergebnis von AiKomPass ist ein persönliches Gesamtprofil, das durch die Angaben der Nutzer individuell erstellt wird. Zu welchem Zweck das Profil eingesetzt wird, ist nicht vorgegeben. Es bestehen vielfältige Möglichkeiten der Nutzung, wobei sich vier Gruppen von Nutzungsmöglichkeiten konkretisieren lassen: die individuelle Nutzung, die betriebliche Nutzung, die außerbetriebliche Nutzung und die Kompetenzanerkennung.

Das im AiKomPass erstellte persönliche Aufgabenprofil kann individuell genutzt werden, um

- selbst einen Überblick über das eigene Wissen und Können zu bekommen;

- mit Vorgesetzten Möglichkeiten einer Veränderung der eigenen Arbeitsaufgaben zu besprechen;

- es einer Bewerbung beizufügen;

- Hinweise zu bekommen, in welchen Bereichen persönlicher Weiterbildungsbedarf besteht.

Betrieblich kann AiKomPass genutzt werden

- im Rahmen von Personal- und Personaleinsatzplanung;

- um Mitarbeitergespräche zu begleiten;

- die Kompetenzen der Mitarbeiter mit den Anforderungen eines Berufsbilds oder eines betrieblichen Kompetenzprofils abzugleichen;

- Weiterbildung und Weiterqualifizierung von Mitarbeitern zu planen und anzustoBen.

Außerbetrieblich kann der AiKomPass genutzt werden für:

- Stellenvermittlung und Arbeitsplatzberatung;

- Reflexion über berufsrelevante Kompetenzen und berufliche Zielsetzungen.

AiKomPass bietet im Rahmen von Kompetenzanerkennung die Möglichkeit,

- die Externenprüfung vorzubereiten;

- durch die Anschlussfähigkeit an Ausbildungsordnungen und Berufsbilder Prüfungsleistungen anzuerkennen;

- eine Gleichwertigkeitsfeststellung von Berufsabschlüssen vorzunehmen;

- in Validierungsverfahren erfasste und bewertete Kompetenzen auf die berufliche Qualifizierung als Teilleistungen anzurechnen;

- Berufs-, Arbeits- und Ausbildungsprofile zu erstellen. 


\section{Diskussion und Ausblick}

In der Testphase und den anschließenden Erprobungen zeigte sich, dass Interessierte (auch ohne formalen Berufsabschluss) AiKomPass nutzen und für sich ein individuelles Kompetenzprofil erstellen können. Ob das ohne jegliche fremde Hilfe gelingt, ist vor allem von der vorhandenen Sprachkompetenz abhängig. Obwohl in AiKomPass keine komplizierten Kompetenzbeschreibungen vorkommen - ganz im Gegenteil -, muss man sich jedoch verdeutlichen, dass AiKomPass ein sprachbasiertes Instrument ist, dessen Benutzung das Verstehen der vorhandenen Arbeitsfeldund Aufgabenbeschreibungen unterstellt.

Im Rahmen des EU-Projektes „Labour INT“ ist AiKomPass bereits ins Englische übersetzt worden. Weitere Sprachen sollen hinzukommen. Hierbei stehen beispielsweise Zugewanderte im Fokus, die sich im Herkunftsland bereits arbeitsrelevantes Wissen und Können angeeignet haben, das sie aber weder formal nachweisen können noch zu beschreiben in der Lage sind. Die ersten Überlegungen und Erfahrungen zeigen jedoch, dass die angestrebte Internationalisierung von AiKomPass kein ausschließlich sprachliches Übersetzungsproblem ist, da Menschen aus anderen Kulturkreisen schon in ihrer Landessprache bisweilen die Worte nicht zur Verfügung haben, die für Aufgabenbeschreibungen in der deutschen Metall- und Elektroindustrie zu verwenden wären. Hier sind daher stärker bild- und grafikbasierte Darstellungen von Aufgabeninventaren hilfreich, die ggf. auch um zusätzliche Erläuterungen ergänzt werden müssten.

Innerhalb der Metall- und Elektroindustrie können auf Basis des vorliegenden Instruments weitere Arbeitsfelder erschlossen werden, wie bspw. kaufmännische Tätigkeitsfelder. Darüber hinaus ist das Aufgabeninventar im Metall- und Elektrohandwerk zu testen und mit entsprechenden Aufgaben zu erweitern. Schließlich kann das dem Kompetenzinstrument AiKomPass zugrunde liegende Konzept auch auf andere Branchen übertragen und ausgeweitet werden.

Die durchweg positive Resonanz der beteiligten Betriebe, Sozialpartner und vor allem - der Arbeitnehmer im Rahmen der Evaluation der Projekte wird von uns als Bestätigung des tätigkeitsbasierten Kompetenzerfassungsinstruments AiKompass betrachtet. Man kann sich jedoch fragen, was solch eine angeleitete Selbsteinschätzung mithilfe von AiKomPass wert ist. Derartige Fragen kamen und kommen vor allem aus der Wissenschaft, während die betrieblichen Praktiker betonen, dass die bislang erstellten Kompetenzprofile eher eine Untertreibung des tatsächlichen beruflichen Könnens einer Person darstellen als eine Übertreibung. Dennoch ist richtig, dass hier wie bei allen Formen der Selbsteinschätzung die Objektivität des Verfahrens fragwürdig ist (Nickolaus et al. 2011, S. 58).

Daher schätzen wir AiKomPass im Wesentlichen als hilfreiches Instrument bei zwei der vier Stufen auf dem Weg zur Validierung informell erworbener Kompetenzen ein (Gutschow und Jörgens 2018, S. 2), nämlich bei der Identifizierung und Dokumentierung arbeitsrelevanter Erfahrungen, und halten AiKomPass für die formale Bewertung und Zertifizierung allein für nicht ausreichend. Jedoch ist AiKomPass anschlussfähig an Fragen der formalen Anerkennung von Berufsabschlüssen. 
In einer Masterarbeit (Köhn 2017) konnte für den Ausbildungsberuf „Elektroniker/-in für Geräte und Systeme“ gezeigt werden, dass mit ganz geringfügigen Modifizierungen AiKomPass in der Lage ist, Entsprechungen zum Ausbildungsrahmenplan abzubilden. Das bedeutet, dass mit einer entsprechenden Anleitung zu ersehen ist, welche Aufgaben jemand im AiKomPass bestätigt haben müsste, damit das hierin zum Ausdruck kommende Können dem Ausbildungsberufsbild vollständig entspricht. Sicherlich: Das Problem bei dieser Art von Kompetenzdiagnostik besteht darin, dass allein schon aufgrund des möglichen Umfangs von beherrschten Arbeitsaufgaben wenig über die Qualität der Aufgabenbearbeitung ausgesagt wird. Aber immerhin werden Angaben zum Umfang eines individuellen Kompetenzprofils gemacht, und dieser Umfang kann mit dem im Ausbildungsberufsbild geforderten Umfang abgeglichen werden, während alle bekannten Verfahren der beruflichen Kompetenzdiagnostik versuchen, aus einem mehr oder minder kleinen Ausschnitt tiefergehend getesteter Kompetenzen Aussagen für die berufliche Gesamtqualifikation abzuleiten. Aussagen über die Breite der bei einer Person vorhandenen Kompetenzen sind gerade bei der formalen Anerkennung informellen Lernens relevant, weil man hier ja - anders als bei Facharbeiterprüfungen und anders als bei Kompetenzdiagnostik innerhalb formaler Bildungsgänge - nicht davon ausgehen kann, dass Anerkennungswillige alle Elemente des Ausbildungsrahmenplans zumindest als Input schon einmal kennengelernt haben. Aussagen über den vorhandenen oder fehlenden Umfang von Kompetenzen auf Basis von AiKomPass stellt auch die Anschlussfähigkeit zur Weiterbildungsplanung her, sodass gezielt Lücken geschlossen werden können (Fischer et al. 2017).

Fazit: Mithilfe von AiKomPass können auch wenig geübte Menschen ein individuelles Kompetenzprofil erstellen, also für sich arbeitsrelevante Kompetenzen identifizieren und dokumentieren. Dieses Kompetenzprofil genügt den testtheoretischen Gütekriterien der Kompetenzdiagnostik allein nicht, ist aber anschlussfähig. Eine der wichtigsten Erkenntnisse aus den Evaluationen und Erprobungen mit AiKomPass war jedoch: Eine angeleitete Selbsteinschätzung kann die Betroffenen selbst zu Akteuren der Kompetenzdiagnostik machen, was ein Stimulus für deren Bildungsaspirationen sein kann (Fischer et al. 2014).

Open Access Dieser Artikel wird unter der Creative Commons Namensnennung 4.0 International Lizenz (http://creativecommons.org/licenses/by/4.0/deed.de) veröffentlicht, welche die Nutzung, Vervielfältigung, Bearbeitung, Verbreitung und Wiedergabe in jeglichem Medium und Format erlaubt, sofern Sie den/die ursprünglichen Autor(en) und die Quelle ordnungsgemäß nennen, einen Link zur Creative Commons Lizenz beifügen und angeben, ob Änderungen vorgenommen wurden.

Publisher's Note Springer Nature remains neutral with regard to jurisdictional claims in published maps and institutional affiliations.

\section{Literatur}

Baethge, M., Brunke, J., \& Wieck, M. (2010). Die Quadratur des Kreises - oder die Mühsal der Suche nach Indikatoren für informelles Lernen: am Beispiel beruflichen Lernens im Erwachsenenalter. In Bundesministerium für Bildung und Forschung (BMBF) (Hrsg.), Indikatorenentwicklung für den nationalen Bildungsbericht (S. 157-190). Bonn, Berlin: Bundesministerium für Bildung und Forschung (BMBF). 
Bauer, H., Böhle, F., Munz, C., Pfeiffer, S., \& Woicke, P. (2002). High-Tech-Gespür. Erfahrungsgeleitetes Arbeiten und Lernen in hoch technisierten Arbeitsbereichen. Bielefeld: W. Bertelsmann.

Bauer, W., Koring, C., Röben, P., \& Schnitger, M. (2007). Abschlussbericht Projekt Weiterbildung im Prozess der Arbeit für Fachkräfte in der Metall- und Elektroindustrie in Baden-Württemberg (WAP). Bremen. http://www.wap.agenturq.de/news/meldung.html?id=167. Zugegriffen: 15. Dez. 2014.

Böhle, F., \& Rose, H. (1992). Technik und Erfahrung. Arbeit in hochautomatisierten Systemen. Frankfurt a. M., New York: Campus.

Boreham, N.C. (2004). Collective competence and work process knowledge. European conference on educational research, University of Crete, Greece. http://citeseerx.ist.psu.edu/viewdoc/download? doi=10.1.1.521.9147\&rep=rep1\&type=pdf. Zugegriffen: 15. März 2018.

Boreham, N.C., Samurcay, R., \& Fischer, M. (Hrsg.). (2002). Work process knowledge. London, New York: Routledge.

CEDEFOP - European Centre for the Development of Vocational Training (2009). European guidelines for validating non formal and informal learning. Luxembourg. http://www.cedefop.europa.eu/en/Files/ 4054_EN.PDF. Zugegriffen: 15. März 2018.

Colley, H., Hodkinson, P., \& Malcolm, J. (2003). Informality and formality in learning. Learning and skills research centre. University of Leeds. http://www.uk.ecorys.com/europeaninventory/publications/ concept/lsrc_informality_formality_learning.pdf. Zugegriffen: 15. März 2018.

Eraut, M., Alderton, J., Cole, G., \& Senker, P. (1998). Development of knowledge and skills in employment. Brighton: University of Sussex, Institute of Education.

Fischer, M. (2000). Von der Arbeitserfahrung zum Arbeitsprozeßwissen. Rechnergestützte Facharbeit im Kontext beruflichen Lernens. Opladen: Leske + Budrich. unv. Neuauflage: Berlin et al.: Springer.

Fischer, M., \& Röben, P. (2004). Arbeitsprozesswissen im Fokus von individuellem und organisationalem Lernen. Ergebnisse aus Großbetrieben in vier europäischen Ländern. Zeitschrift für Pädagogik, 50(2), 182-201.

Fischer, M., Boreham, N.C., \& Nyhan, B. (Hrsg.). (2004). European perspectives on learning at work: the acquisition of work process knowledge. Cedefop reference series. Luxembourg: Office for Official Publications for the European Communities.

Fischer, M., Huber, K., Mann, E., \& Röben, P. (2014). Informelles Lernen und dessen Anerkennung aus der Lernendenperspektive - Ergebnisse eines Projekts zur Anerkennung informell erworbener Kompetenzen in Baden-Württemberg. In bwp@Berufs- und Wirtschaftspädagogik - online, 26, 1-21. http:// www.bwpat.de/ausgabe26/fischer_etal_bwpat26.pdf. Zugegriffen: 15. März 2018.

Fischer, M., Röben, P., \& Sandal, C. (2017). Erfassung informell erworbener Kompetenzen und Weiterbildungsplanung für Geringqualifizierte auf Basis von AiKomPass. In B. Matthes \& E. Severing (Hrsg.), Berufsbildung für Geringqualifizierte - Barrieren und Erträge (S. 111-130). Bielefeld: W. Bertelsmann.

Frieling, E., Kauffeld, S., \& Grote, S. (2000). Fachlaufbahnen für Ingenieure - Ein Vorgehen zur systematischen Kompetenzentwicklung. Zeitschrift für Arbeitswissenschaft, 54, 165-174.

Gutschow, K., \& Jörgens, J. (2018). Herausforderungen bei der Einführung von Verfahren zur Validierung nicht formalen und informellen Lernens in Deutschland. BIBB Report, 1.

Holzkamp, K. (1993). Lernen. Subjektwissenschaftliche Grundlegung. Frankfurt a. M., New York: Campus.

Köhn, D. (2017). Sichtbarmachung von beruflichem Können. Untersucht anhand einer dokumentenbasierten Inhaltsanalyse am Beispiel des Tätigkeitsinventares von AiKomPass und des Ausbildungsrahmenplans für Elektroniker für Geräte und Systeme. Karlsruhe: Karlsruher Institut für Technologie, Institut für Berufspädagogik und Allgemeine Pädagogik. Masterarbeit

Lipsmeier, A. (1996). Lernen und Arbeiten. In B. Bonz (Hrsg.), Didaktik der Berufsbildung (S. 205-219). Stuttgart: Holland \& Josenhans.

Nickolaus, R., Gschwendtner, T., \& Abele, S. (2011). Valide Abschätzungen von Kompetenzen als eine notwendige Basis zur Effektbeurteilung pädagogischer Handlungsprogramme - Herausforderungen, Ansätze und Perspektiven. In M. Fischer, M. Becker \& G. Spöttl (Hrsg.), Kompetenzdiagnostik in der beruflichen Bildung - Probleme und Perspektiven (S. 56-74). Frankfurt a. M.: Peter Lang.

OECD - Organisation for Economic Co-operation and Development (2006). New OECD activity on recognition of non-formal and informal learning. Guidelines for country participation. http://www.oecd. org/edu/skills-beyond-school/recognitionofnon-formalandinformallearning-home.htm. Zugegriffen: 15. März 2014

Reinhold, M., Haasler, B., Howe, F., Kleiner, M., \& Rauner, F. (2003). Curriculum Design II: Entwickeln von Lernfeldern. Von beruflichen Arbeitsaufgaben zum Berufsbildungsplan. Konstanz: Christiani.

Schier, F. (2011). Übergänge ins Beschäftigungssystem - Herausforderungen an der ersten und zweiten Schwelle. In bwp@ Spezial 5 - Hochschultage Berufliche Bildung 2011, Fachtagung 15 (S. 1-14), 
hrsg. v. E. Jung, M. Kenner \& H.-G. Lambertz. http://www.bwpat.de/ht2011/ft15/schier_ft15-ht2011. pdf. Zugegriffen: 15. März 2018.

Schön, D. A. (1983). The reflective practitioner. How professionals think in action. New York: Basic Books. Siebeck, F. (1994). Arbeitserfahrungen und Erfahrungslernen - was ist das? In K. Drechsel, P. Storz \& G. Wiesner (Hrsg.), Gestaltungsperspektiven der Chemiearbeit in Produktion und Labor (S. 100-124). Hamburg, Dresden: VMS.

Südwestpresse (2014). Neuer BA-Chef will Ungelernte ausbilden. (Vom 28.08). http://www.swp.de/ulm/ nachrichten/wirtschaft/Neuer-BA-Chef-will-Ungelernte-ausbilden;art4325,2766889. Zugegriffen: 15. Sept. 2014.

Witzgall, E. (2009). Kompetenzmanagement in der industriellen Produktion. Das Tool CM ProWork. Renningen: Expert. 